\title{
Real-time ultrasound-guided vs. anatomical landmark-guided paramedian epidural anesthesia in overweight parturients undergoing analgesic labor: a randomized controlled trial
}

\author{
Jindi Jiang ${ }^{1}$, Yujie $\mathrm{Xu}^{1}$, Mingfeng $\mathrm{He}^{1, *}$
}

\author{
${ }^{1}$ Department of Anesthesiology and \\ Perioperative Medicine, The First \\ Affiliated Hospital of Nanjing Medical \\ University, 210029 Nanjing, Jiangsu, \\ China
}

\section{*Correspondence}

18551682286@126.com

(Mingfeng $\mathrm{He}$ )

\begin{abstract}
We evaluated whether real-time ultrasound-guided epidural block is more suitable for overweight parturients undergoing analgesic labor than traditional palpation positioning. Sixty overweight at-term pregnant women (body mass index $\geq 30 \mathrm{~kg} / \mathrm{m}^{2}$ ) with singleton pregnancy, prepared for vaginal delivery with epidural analgesia, were randomly allocated into two groups. The parturients in the anatomical landmark catheterization group received paramedian epidural anesthesia using the anatomical landmarkguided technique, while real-time ultrasound-guided positioning was performed in the ultrasound-guided-catheterization group. Total procedure duration, time to identify the puncture site and perform the puncture, first attempt success rate, number of attempts, number of needle-redirections, intervertebral-space-change rate, satisfaction score, and complications were compared between the groups. Procedure duration and time to identify the puncture site were significantly shorter in the anatomical landmark catheterization group $(440.1 \pm 97.2 \mathrm{~s}$ vs. $521.9 \pm 68.4 \mathrm{~s}, p<0.001$ and $24.9 \pm$ $13.6 \mathrm{~s}$ vs. $112.2 \pm 15.6 \mathrm{~s}, p<0.001$, respectively). There was no significant difference in the time to perform the puncture $(385.3 \pm 89.7 \mathrm{~s}$ vs. $365.1 \pm 73.0 \mathrm{~s}, p$ $=0.341$ ). The first attempt success rate was lower while the number of attempts and number of needle-redirections were higher in the anatomical landmark catheterization group $(p<0.05)$. The intervertebral-space-change rate was similar across the groups. Satisfaction was significantly lower in the ultrasound-guided catheterization group ( $p$ $=0.009$ ). Complication occurrence, e.g., catheterization difficulty or bleeding during catheterization, dural puncture, and lower-back pain, was similar across the groups. Real-time ultrasound-guided paramedian epidural anesthesia improved the first attempt success rate and reduced the number of attempts and number of needle-redirections in overweight parturients undergoing analgesic labor. However, the longer total procedure duration and time to identify the puncture site might dissatisfy parturients.
\end{abstract}

\section{Keywords}

Real-time ultrasound-guided; Paramedian epidural block; Overweight; Parturients; Analgesic labor

\section{Introduction}

It was estimated that more than half of all pregnant women in the United States were overweight in 2011, and the prevalence has been increasing since [1]. According to the 2015 International Federation of Gynecology and Obstetrics report, obesity increases health risks for the mother and fetus and makes epidural anesthesia more difficult to administer [2].

Neuraxial anesthesia is considered the best technique for women undergoing delivery who request labor analgesia, as it provides satisfactory analgesic effects. However, it is more difficult to perform epidural puncture in parturients with pregnancy-induced ligament softening and increased adipose tissue [3]. Since it is a relatively blind procedure, vertebral localization based on anatomical landmarks is often erroneous, and the "loss-of-resistance" method might lead to incorrect insertion depth, especially in overweight patients [4].

A growing number of studies in recent years have begun reporting epidural anesthesia under ultrasound guidance. Administering ultrasound-guided epidural anesthesia is a relatively easy procedure, and it can decrease the number of attempts to access the epidural space, the level of periprocedural pain, and discomfort scores $[5,6]$. 
Research has shown that pre-puncture ultrasound-guided epidural anesthesia has significant benefits for parturients [7]. However, the effect of real-time ultrasound-guided epidural anesthesia on overweight parturients remains unclear. This study was designed to determine if real-time ultrasoundguided epidural block is more suitable for overweight parturients undergoing analgesic labor than the traditional palpation-positioning method.

\section{Materials and methods}

\subsection{Ethical approval and clinical trial registry}

This was a prospective, randomized controlled trial. The Ethics Committee of the First Affiliated Hospital of Nanjing Medical University approved this study (no. 2020-SR-515, December 30, 2020). The study was registered in the Chinese Clinical Trial Registry (no. ChiCTR2100042791, January 28, 2021). All participants provided written informed consent.

\subsection{Recruitment and randomization}

This study is part of a series entitled "Application of real-time ultrasonic-guided epidural puncture in overweight parturient women and its effect on labor analgesia". The initial study plan was to compare two anatomical landmark-guided catheterization and two ultrasound-guided catheterization methods commonly used in clinical practice. However, during the preexperiment, we found that the parturients seemed to show more dissatisfaction with real-time ultrasound-guided punctures, despite the higher first attempt success rate and fewer attempts. This drew our attention. Therefore, we used the total duration of the operation, the greatest maternal concern, as the primary observation, recalculated the sample size based on the preexperiment data, and reported this part of the study in advance.

Of the 65 parturients assessed for eligibility between February 1, 2021 and 15 March, 2021, we recruited 60 overweight parturients (body mass index $[\mathrm{BMI}] \geq 30 \mathrm{~kg} / \mathrm{m}^{2}$ ) with singleton pregnancy, prepared for vaginal delivery, who requested epidural analgesia during labor at the First Affiliated Hospital of Nanjing Medical University. The exclusion criteria were: contraindications to epidural puncture, central nervous system disease, a history of lumbar surgery or trauma, scoliosis, and refusal to cooperate or participate in the study.

The recruited parturients were randomly assigned into two groups, 30 in each, using a computer-generated random number table. Characteristics of the subjects, including age, BMI, American Society of Anesthesiologists [ASA] score, and abdominal girth (measured at the level of the umbilicus in the transverse plane while the parturients were in a supine position) were recorded for analysis. The parturients in the anatomical landmark catheterization (ALC) group received paramedian epidural anesthesia using the anatomical landmark-guided technique, while real-time ultrasoundguided positioning was performed in the ultrasound-guided catheterization (UGC) group. The anesthesiologist and data collectors were not informed of the parturient grouping until all base value measurements were performed.

\subsection{Anesthesia procedure and real-time ultrasonographic scanning}

This current study focuses on the comparison of two groups in the series, real-time ultrasound-guided versus anatomical landmark-guided paramedian epidural anesthesia, to demonstrate our findings in the pre-experiment.

Pulse oxygen content, electrocardiography, and noninvasive blood pressure were routinely monitored in all parturients, followed by epidural anesthesia in the lateral decubitus position. Epidural nerve block anesthesia was performed by the same anesthesiologist who had worked in the Department of Anesthesiology for more than 8 years and had performed more than 50 ultrasound-guided neuraxial anesthesia procedures.

The palpation technique was used to locate the intervertebral space in the ALC group. The intersecting point between the spine and the highest point of the iliac crest on both sides was defined as the L3-4 intervertebral space or the L4 spinous process of the vertebral body (Fig. 1a). Subsequently, ultrasonography was used to evaluate the palpation method positioning accuracy. The positioning result was not revealed to the anesthesiologist performing the procedure, and the site determined by palpation was used as the puncture site. The time required for the ultrasonographic evaluation was deducted from the total procedure duration. he midpoint of the selected space was positioned and marked as the puncture point with a skin-marking pen, and the puncture direction was determined by experience (Fig. 1b).

The position of the intervertebral spaces in the UGC group was clarified by ultrasonography. A curved probe (C5-2, 5$2 \mathrm{MHz}$ ) and a Compass ultrasound system (Huasheng, Inc., Shenzhen, Guangdong, China) were used. After proper sterilization, the ultrasound gel was smeared on the probe, and the transducer and cable were covered with a sterile plastic sleeve. The probe was placed 1-2 cm lateral to the long axis of the spine with the orientation marker directed cranially and tilted slightly medially to allow the ultrasound beam to enter the spinal canal unimpeded (Fig. 2a). While maintaining the same orientation, the transducer was moved caudally to identify the sacrum. The interlaminar space on the cephalic side of the sacrum was considered as the L5/S1 intervertebral space. Other intervertebral spaces were identified by counting them one by one.

The position and direction of the ultrasonographic probe were adjusted to ensure that the L3/L4 intervertebral space was displayed as clearly as possible and positioned at the center of the image (Fig. 2b). Local infiltration was performed by subcutaneous injection of $2-3 \mathrm{~mL}$ of $2 \%$ lidocaine $1-2 \mathrm{~cm}$ caudal to the probe, and the point of infiltration was selected as the epidural puncture point. Subsequently, the ultrasound probe was held by the operator in the left hand while the puncture was performed with the right hand. In recent years, this real-time ultrasound-guided puncture method has been reported by several institutes $[5,8]$, and it is also the method commonly used in our department. During the puncture, a nurse unrelated to the study was simultaneously wearing gloves and giving the necessary assistance when needed. The needle direction was adjusted under ultrasound guidance and 

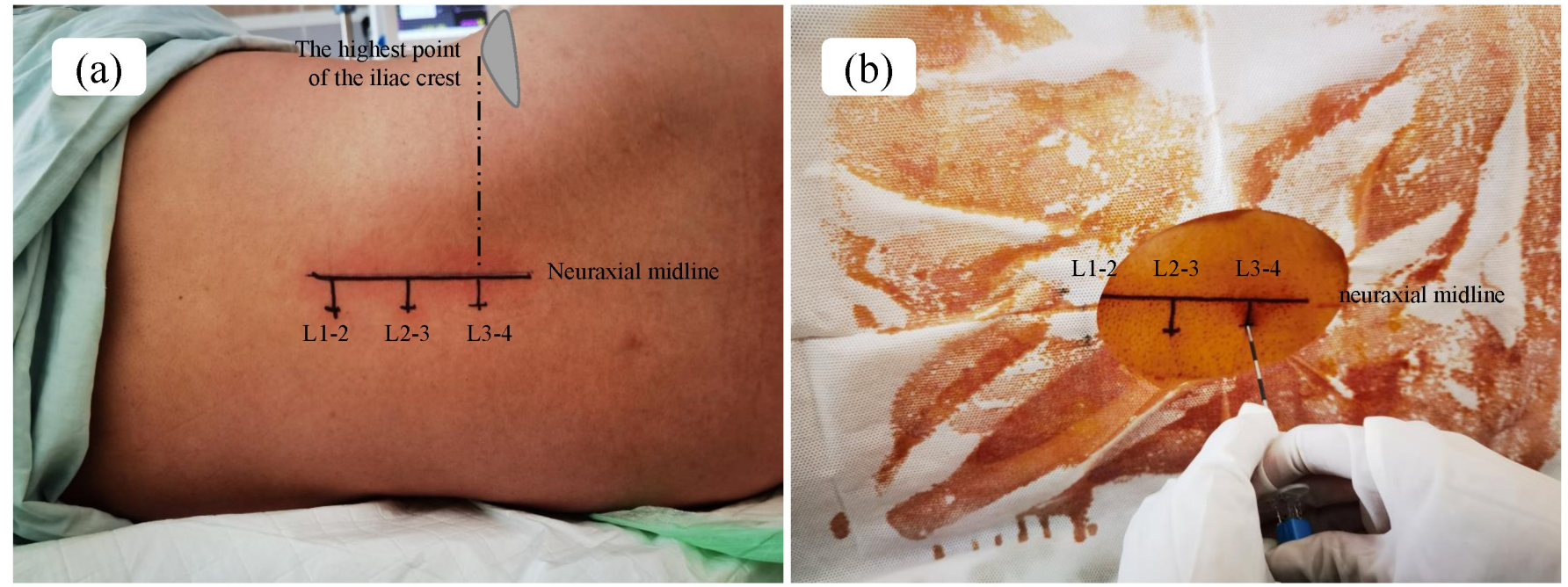

F I G U RE 1. Skin markings and needle insertion based on experience. (a) Using the palpation technique to locate the intervertebral space in the ALC group. (b) Paramedian needle insertion based on experience.
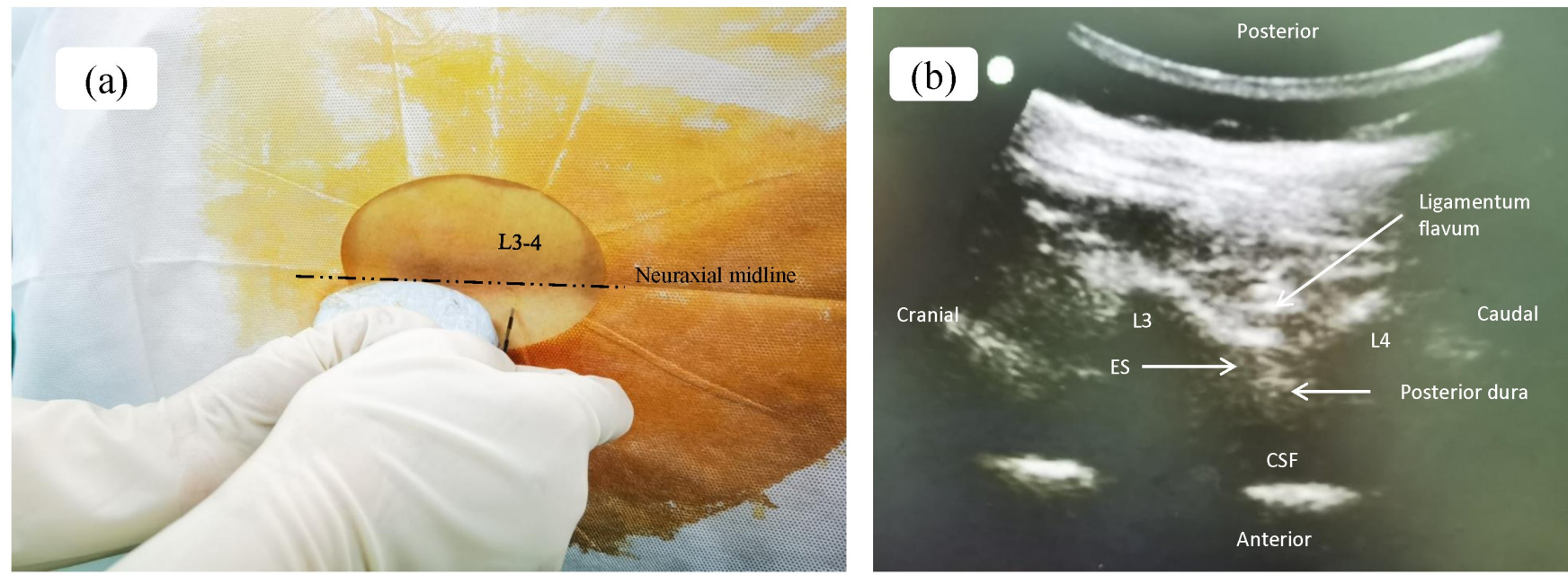

F I G U R E 2. Ultrasound-guided insertion and ultrasound imaging. (a) Ultrasound-guided paramedian epidural anesthesia. (b) Paramedian sagittal view. ES, epidural space; CSF, cerebrospinal fluid.

advanced slowly into the target interlaminar space in a needlein-plane manner. When the needle tip was visualized or estimated (when the needle or its tip were not clearly visible) to have reached the epidural space, the resistance-to-air method was used to confirm the localization.

The local anesthetic used for subcutaneous infiltration anesthesia (2-3 mL of $2 \%$ lidocaine) and test dose (to test whether the needle tip accidentally entered a blood vessel or the subarachnoid space, $3 \mathrm{~mL}$ of $2 \%$ lidocaine) was the same in both groups. After a successful puncture, a catheter was inserted into the epidural space through a Tuohy needle and connected to an analgesia pump with $124 \mathrm{~mL}$ of $0.121 \%$ ropivacaine and $1.67 \mu \mathrm{g} / \mathrm{mL}$ fentanyl. The analgesia pump was set to deliver a loading dose of $10 \mathrm{~mL}$, followed by a continuous infusion rate of $9 \mathrm{~mL} / \mathrm{h}$, a self-controlled volume of $5 \mathrm{~mL} /$ bolus, and a locking time of $15 \mathrm{~min}$.

\subsection{Blindness}

Throughout the procedure and postoperative follow-up, the parturients had been blinded to group allocation to avoid subjective perception bias, while the anesthesiologist in charge of the puncture procedure was informed of the group allocation. The results were evaluated by two independent observers. One observer, who was responsible for the total procedure duration, time to identify the puncture site and perform the puncture, and first attempt success rate, was referred to as the primary observer and was informed of group allocation due to the need to observe the puncture procedure. The other observer, who was responsible for assessment of anesthesia effect, periprocedural patient discomfort scores, and anesthesia complications, was blinded to group allocation.

\subsection{Data collection and analysis}

The primary outcome of the study was the total procedure duration, which would reflect the difficulty and feasibility of 
both techniques [8].

The secondary outcomes included:

(1) Time to identify the puncture site and perform the puncture.

(2) Puncture accuracy: positioning accuracy (parturients in the ALC group), first attempt success rate (success rate on first attempt to access the epidural space without redirection), number of attempts (a new attempt refers to reinsertion after removal of the needle from the skin), number of needle-redirections (backward movement of the puncture needle without exiting the skin), and change of the vertebral level.

(3) Anesthesia effect: the level of sensory loss at 10, 15, and $30 \mathrm{~min}$ after initial anesthesia administration (tested by loss of cold sensation).

(4) Periprocedural patient discomfort scores: the scores were obtained using a numeric rating scale and are expressed as absolute numbers. The values were graded on a scale from 0 to 10: $0=$ dissatisfied, $1-3=$ not very satisfied, $4-6=$ moderately satisfied, and 7-10 = very satisfied. When the discomfort score was below 7, the most unsatisfactory aspect of the puncture procedure would be inquired and recorded.

(5) Complications of anesthesia: paraesthesia or bleeding during catheterization, difficulty in catheterization, dural puncture, and lower back pain.

\subsection{Statistical analysis}

IBM SPSS Statistics for Windows, Version 23.0 (IBM Corp., Armonk, NY, USA) was used for statistical analysis. The Kolmogorov-Smirnov test was used to assess the distribution normality. Values with a normal distribution (age, BMI, abdominal girth, total procedure duration, time to identify the puncture site, and time to perform the puncture) are presented as mean \pm standard deviation ( $\bar{x} \pm \mathrm{SD}$ ) and compared with the Student's $t$-test. Categorical data were analyzed using the chisquared test (first attempt success rate), chi-squared analysis with Yate's correction (change in vertebral level, paresthesia or bleeding during catheterization, difficulty in catheterization), or Fisher's exact test (positioning accuracy, dural puncture, lower back pain). Rank data were analyzed with the Wilcoxon rank-sum test (ASA score, number of attempts, number of needle-redirections, periprocedural patient discomfort score, number of parturients dissatisfied with prolonged procedure duration, and level of sensory loss). Differences were considered statistically significant at $p<0.05$.

After reviewing the relevant literature, we found that the population selection and the research methods were not identical across studies, with sample sizes ranging from 10 to 55 per group $[5,7,8]$. Therefore, in the initial study plan, we set a sample size of 50. After ethics committee review, we conducted the corresponding pre-experiment and recalculated the minimum sample size based on the results. The sample size was based on data from clinical observations of 10 parturients in each group. In this pre-experiment, the total procedure duration in the UGC group was 411, 678, 681, 523, 493, 561, $445,516,526,569(\bar{x} \pm \mathrm{SD}=540.3 \pm 87.6)$ and 325,582 , 411, 484, 556, 308, 660, 398, 475, $397(\bar{x} \pm \mathrm{SD}=459.6 \pm$ 113.7) in the ALC group, respectively. The mean difference between the UGC group and the ALC group was 80.7 (540.3459.6), and the pooled SD was 101.5. To achieve a power of 0.8 and an alpha error less than $0.05,25$ patients were needed in each group. To allow for dropouts, we randomly assigned 30 patients to each group.

\section{Results}

Of 65 patients assessed as eligible, 60 patients were randomized and completed the study. Two patients were excluded because of slight abnormalities in coagulation and three others were excluded due to refusal to participate in the study (Fig. 3). The age, BMI, ASA classification, and abdominal girth of the patients were similar in the two groups (Table 1).

\section{TA B L E 1. Comparison of parturient characteristics} between the two groups ( $n=30$ /group).

$\begin{array}{lccc}\text { Variable } & \text { ALC group } & \text { UGC group } & p \text {-value } \\ \text { Age (years) } & 28.4 \pm 3.4 & 29.2 \pm 3.1 & 0.307 \\ \text { BMI }\left(\mathrm{kg} / \mathrm{m}^{2}\right) & 35.2 \pm 2.4 & 35.6 \pm 2.0 & 0.473 \\ \text { ASA (I/II/III) } & 10 / 18 / 2 & 7 / 21 / 2 & 0.459 \\ \text { Abdominal girth }(\mathrm{cm}) & 119.2 \pm 4.8 & 118.4 \pm 5.0 & 0.496\end{array}$

The age, BMI, and abdominal girth values are expressed as $\bar{x} \pm S D$. The ASA classification grades are expressed as absolute numbers. BMI, body mass index; ASA, American Society of Anesthesiologists; $\bar{x}$, mean; $S D$, standard deviation; $A L C$, anatomical landmark catheterization; UGC, ultrasoundguided catheterization.

The total procedure duration was significantly shorter in the ALC group than the UGC group $(440.1 \pm 97.2 \mathrm{~s}$ vs. 521.9 $\pm 68.4 \mathrm{~s}, p<0.001)$. The time-to-identify the puncture site was also significantly shorter in the ALC group $(24.9 \pm 13.6 \mathrm{~s}$ vs. $112.2 \pm 15.6 \mathrm{~s}, p<0.001)$. The time taken to perform the puncture was similar in both groups, with $385.3 \pm 89.7 \mathrm{~s}$ in the ALC group and 365.0 $\pm 73.0 \mathrm{~s}$ in the UGC group (Table 2).

The first attempt success rate was lower while the number of attempts and number of needle-redirections were higher in the ALC group $(p<0.05)$. To access the epidural space successfully, we had to perform the puncture at the lower L4/L5 intervertebral space in three ALC parturients (because of puncture difficulty rather than positioning failure) and one UGC parturient (unclear ultrasonographic imaging of the Tuohy needle due to uncontrollable movement of the parturient). The rate of change in the vertebral level was similar in the two groups (Table 2).

Interestingly, the satisfaction score in the UGC group was significantly lower than that in the ALC group (Table 3).

Interestingly, the satisfaction score in the UGC group was significantly lower than that in the ALC group. In the ALC group, 6 patients were dissatisfied with the prolonged puncture time, one patient was moderately dissatisfied, and another 5 patients were mildly dissatisfied. While in the UCG group, 13 patients were dissatisfied with the prolonged puncture time, 2 patients were very dissatisfied, 10 patients were moderately dissatisfied, and another 1 was mildly dissatisfied. The difference between the two groups was statistically significant 


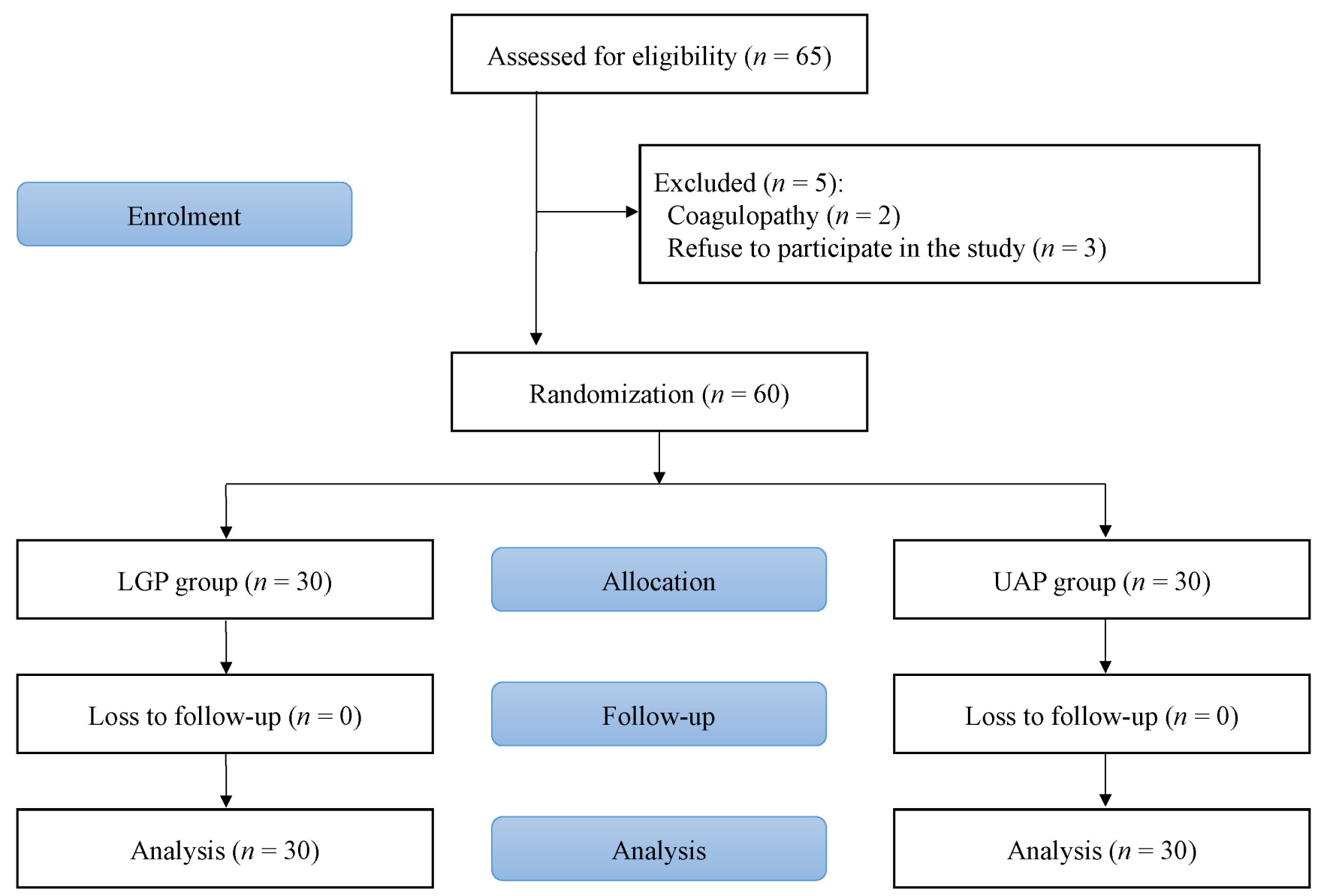

F I G U R E 3. Enrolment, allocation, follow-up and analysis of the study. Flow diagram of the study according to CONSORT 2010 guidelines ALC, anatomical landmark catheterization; UGC, ultrasound-guided catheterization.

TA B L E 2. Comparison of primary and secondary outcomes of parturients in both groups.

\section{Puncture accuracy}

Total procedure duration (s)

Time of puncture site identification (s)

Time to perform the puncture (s)

First attempt success rate

Number of attempts $(1 / 2 / 3)$

Number of needle-redirections $(0 / 1 / 2 / 3 / 4 / 5 / 6)$

Change of the vertebral level

Positioning accuracy

Periprocedural parturients discomfort score (0/1-3/4-6/7-10)

Number of parturients dissatisfied with prolonged procedure duration $(0 / 1-3 / 4-6)$

$\begin{array}{ccc}\text { ALC group } & \text { UGC group } & p \text {-value } \\ 440.1 \pm 97.2 & 521.9 \pm 68.4 & <0.001 * \\ 24.9 \pm 13.6 & 112.2 \pm 15.6 & <0.001 * \\ 385.3 \pm 89.7 & 365.1 \pm 73.0 & 0.341 \\ 9 / 30(30 \%) & 17 / 30(56.7 \%) & 0.037 * \\ 21 / 7 / 2 & 27 / 3 / 0 & 0.048 * \\ 9 / 6 / 7 / 4 / 1 / 1 / 2 & 17 / 9 / 2 / 1 / 1 / 0 / 0 & 0.006 * \\ 3 / 30(10 \%) & 1 / 30(3.33 \%) & 0.605 \\ 25 / 30(86.7 \%) & 30 / 30(100 \%) & 0.052 \\ 3 / 3 / 20 / 4 & 2 / 15 / 12 / 1 & 0.009 * \\ 0 / 1 / 5 & 2 / 10 / 1 & 0.001 *\end{array}$

$A L C$, anatomical landmark catheterization; UGC, ultrasound-guided catheterization. Values of total procedure duration, time to puncture site identification, and time to perform the puncture are expressed as mean and standard deviation. The values of number of needle-redirections and number of attempts are expressed as absolute numbers. First attempt success rate, change in the vertebral level, and positioning accuracy are presented as rates. Periprocedural parturients discomfort scores and number of parturients dissatisfied with prolonged procedure duration were obtained by a numeric rating scale and are expressed as absolute numbers. The numbers in parentheses represent the number of very dissatisfied/moderately dissatisfied/mildly dissatisfied/satisfied parturients or very dissatisfied/moderately dissatisfied/mildly dissatisfied parturients. The values were graded on a scale of 0 to 10: $0=$ very dissatisfied, 1-3 = moderate dissatisfied, $4-6=$ mild dissatisfied, and 7-10 = satisfied. * $p$ $<0.05$. 
TA B L E 3. Comparison of anesthesia effect and complications in the two groups.

\begin{tabular}{|c|c|c|c|}
\hline Parameter & ALC group & UGC group & $p$-value \\
\hline \multicolumn{4}{|l|}{ Level of sensory loss } \\
\hline SL-10 (T7-8/T9-10/T11-12) & $0 / 25 / 5$ & $0 / 26 / 4$ & 0.720 \\
\hline SL-15 (T7-8/T9-10/T11-12) & $18 / 12 / 0$ & $21 / 9 / 0$ & 0.421 \\
\hline SL-30 (T7-8/T9-10/T11-12) & $30 / 0 / 0$ & $30 / 0 / 0$ & 1.000 \\
\hline \multicolumn{4}{|l|}{ Complications } \\
\hline Paraesthesia during catheterization & $6 / 30(20 \%)$ & $1 / 30(3.3 \%)$ & 0.108 \\
\hline Difficulty in catheterization & $7 / 30(23.3 \%)$ & $4 / 30(13.3 \%)$ & 0.505 \\
\hline Bleeding during catheterization & $5 / 30(16.7 \%)$ & $4 / 30(13.3 \%)$ & 1.000 \\
\hline Dural puncture & $2 / 30(6.7 \%)$ & $0 / 30(0 \%)$ & 0.492 \\
\hline Lower back pain & $1 / 30(3.3 \%)$ & $0 / 30(0 \%)$ & 1.000 \\
\hline
\end{tabular}

$(p=0.009$, Table 3$)$. The remaining patients' dissatisfaction with the procedure included repeated punctures, continued contraction pain, concerns about prolonged labor, back pain after labor, and nonspecific reasons.

We evaluated the accuracy of palpation in determining the vertebral space in the ALC group using ultrasonographic scanning. The estimated position was incorrect in five parturients (success rate of $86.7 \%$ ).

The values for sensory loss values are expressed as the number of parturients with sensory loss, tested with cold sensation at the T7-T8, T9-T10, and T11-T12 planes 10, 15, and $30 \mathrm{~min}$ after the initial anesthetic administration [9]. The anesthesia effect at each point and time point was similar in the two groups. No patient underwent cesarean section due to malposition or malpresentation of the fetus, poor anesthetic effect, or other reasons (Table 3).

Six patients in the ALC group, and only one in the UGC group, experienced paresthesia or electric shock-like sensation during catheterization. However, the difference was insignificant $(p=0.108)$. Complications such as difficulty in catheterization or bleeding during catheterization, dural puncture, and lower back pain were also similar. No patients experienced numbness of the lower extremities, headache, or any other side effects.

\section{Discussion}

The current study shows that real-time ultrasound-guided catheterization technology reduces the technical difficulty of epidural anesthesia in overweight parturients undergoing analgesic labor. Compared with the ALC group, the first attempt success rate was higher while the number of attempts and number of needle-redirections were lower in the UGC group. Unexpectedly, maternal satisfaction with the procedure was lower in the UGC group, possibly due to the longer total procedure duration and the time needed to identify the puncture site.

Overweight parturients have slower cervical dilatation rates
[10] and longer latent phase and total labor duration than normal-weight parturients [11]; therefore, they may experience more pain during childbirth and have a more urgent need for pain relief. However, epidural anesthesia is particularly difficult to administer to obese patients because the anatomical landmarks are obscured by adipose tissue and pregnancyinduced softening of the soft tissues and ligaments [2, 4].

Ultrasonography has been widely used in obstetric anesthesia in recent years; however, real-time ultrasonography experience is relatively limited. This study was designed to test whether real-time ultrasound-guided epidural block was more suitable for overweight parturients undergoing analgesic labor than the traditional palpation-positioning method.

Because of concerns regarding prolongation of the labor process [12] or reduction of uterine contractility [13], epidural puncture and catheterization were performed at our institution for parturients in need of epidural analgesia for pain relief only when the cervical dilation was approximately $2-3 \mathrm{~cm}$. Parturients often feel a certain degree of pain at this point and wish to relieve it as soon as possible. For this reason, we chose the total procedure duration as the primary outcome. Furthermore, this parameter could reflect the ease and feasibility of the techniques [8]. Ultrasonography can accurately estimate the depth of the epidural space, but using it requires much time [14]. Our results showed that the total procedure duration and time to identify the puncture site in the UGC group were significantly greater than those in the ALC group. These findings were similar to those of Park et al. [9] who studied older people, in whom the mean identifying and total procedure times were $17.5 \mathrm{~s}$ vs. $117.5 \mathrm{~s}$ and $92.5 \mathrm{~s}$ vs. $181.5 \mathrm{~s}$ in the anatomical landmark-guided group and the ultrasoundassisted technique group.

Nassar and Abdelazim [7] compared the pre-puncture ultrasound-guided epidural insertion and palpation methods before vaginal delivery. Their results showed a higher first attempt success rate and fewer attempts in the ultrasound-guided group. Vallejo et al. [15] also showed that ultrasonographic measurement of the epidural space depth 
decreased the failed epidural puncture rate and the number of attempts in resident trainees. The results of the current study were similar, but differed in that the puncture was not measured before the procedure, but performed under realtime ultrasound guidance. As we know, the angle between ultrasound beam and skin is difficult to measure accurately, and the subsequent puncture is also difficult to match exactly the path of the previous examination. Therefore, the real-time guidance technology used in this study can provide higher accuracy $[5,8]$. In our study, the first attempt success rate, number of attempts, and number of needle-redirections in the UGC group were significantly lower than those in the ALC group, but the rate of vertebral level change was similar in both groups, concurring with the results of Nassar [7] and Vallejo et al. [15].

Although the palpation positioning accuracy in our study was only $86.7 \%$, the sensory loss level was similar in both groups at each time point. Grau et al. [16] suggested that ultrasound-guided epidural anesthesia was well-received by pregnant women. We expected parturients in the UGC group to show higher satisfaction levels because of the higher positioning accuracy ( $25 / 30$ vs. $30 / 30$ parturients, although the difference was statistically insignificant) and first attempt success rate and fewer attempts and number of needle-redirections. Interestingly, the opposite was true. The procedure length, rather than puncture accuracy, had the prime impact on the parturient satisfaction in the UGC group when interviewed during a follow-up meeting after delivery. This remained unchanged even if we informed them that parturients in the ALC group might have experienced different pain types owing to the lower first attempt success rate, more attempts, and number of needle-redirections. This finding was inconsistent with the findings of Lim et al. [17] and Wang et al. [18]. This difference was probably because our patients were overweight parturients with difficulty in epidural puncture and had varying degrees of labor pain when the procedure was performed. The main complaint in the UGC group was the prolonged operation time. Six parturients in the ALC group and 13 in the UGC group complained of the procedure length. Several parturients recalled unbearable labor pains during the puncture process. The time to perform the puncture was similar in both groups; however, in the ALC group 511, 542, and $611 \mathrm{~s}$ were needed to access the epidural space in three parturients, which was much longer than the time needed in the UGC group (223-487 s). The main cause of dissatisfaction in the ALC group was repeated punctures, especially in the three patients mentioned above. Eight parturients in the ALC group and one in the UGC group complained of repeated punctures.

The present study has certain limitation. This was a singlecentered research, and the proficiency of the anesthesiologist who performed the procedure cannot be regarded as a general representative, which may be a potential source of bias. However, as mentioned above, the results are similar to many previous researches, so the research is representative to some extent.

Based on the nature of the study, neither the operator nor the primary observer could be blinded to group allocation. During the procedure, the operator in the GUC group must hold the probe in the left hand and perform the puncture with the right hand. The stability and accuracy of the puncture would be reduced when the operator wasn't proficient in the technique.

In addition, we modified the initial protocol based on the results of the pre-experiment to include total procedure duration as the primary outcome and recalculated the sample size, which is also one of the limitations of the study. Finally, the sample size of this study was small, and therefore, more studies with larger samples are needed to further discuss the issue.

\section{Conclusions}

In summary, our study revealed that real-time ultrasoundguided paramedian epidural anesthesia improved the first attempt success rate and reduced the number of attempts and number of needle-redirections in overweight parturients undergoing analgesic labor compared to the traditional palpation method. However, parturients were dissatisfied with the longer total procedure duration, and the time needed to identify the puncture site.

\section{AUTHOR CONTRIBUTIONS}

JJ-Conception and design, Provision of study materials or patients, Data collection or management, Manuscript writing, Final approval. $\mathrm{MH}$ - Conception and design, Administrative support, Data collection and management, Data analysis and interpretation, Manuscript writing, Final approval. YXProvision of study materials or patients, Data analysis and interpretation, Manuscript writing, Final approval.

\section{ETHICS APPROVAL AND CONSENT TO PARTICIPATE}

The trial was conducted in accordance with the Declaration of Helsinki (as revised in 2013). The study was approved by the Ethics Committee of the First Affiliated Hospital of Nanjing Medical University (NO.: 2020-SR-515, on December 30, 2020).

\section{ACKNOWLEDGMENT}

We would like to thank Editage (www.editage.cn) for English language editing.

\section{FUNDING}

This research received no external funding.

\section{CONFLICT OF INTEREST}

The authors declare no conflict of interest.

\section{CONSENT TO PARTICIPATE}

All individual participants provided written informed consent. 


\section{AVAILABILITY OF DATA AND MATERIAL}

The data are available from the corresponding author on reasonable request.

\section{CONSENT FOR PUBLICATION}

Not applicable.

\section{REFERENCES}

[1] Ogden CL, Carroll MD, Fryar CD, Flegal KM. Prevalence of obesity among adults and youth: United States, 2011-2014. NCHS Data Brief. $2015 ; 1-8$.

[2] Taylor CR, Dominguez JE, Habib AS. Obesity and obstetric anesthesia: current insights. Local and Regional Anesthesia. 2019; 12: 111-124.

[3] Buddeberg BS, Bandschapp O, Girard T. Post-dural puncture headache. Minerva Anestesiologica. 2019; 85.

[4] Mace HS, Paech MJ, Mcdonnell NJ. Obesity and Obstetric Anaesthesia. Anaesthesia and Intensive Care. 2011; 39: 559-570.

[5] Grau T, Leipold RW, Fatehi S, Martin E, Motsch J. Real-time ultrasonic observation of combined spinal-epidural anaesthesia. European Journal of Anaesthesiology. 2004; 21: 25-31.

[6] Grau T, Leipold RW, Horter J, Conradi R, Martin E, Motsch J. The lumbar epidural space in pregnancy: visualization by ultrasonography. British Journal of Anaesthesia. 2001; 86: 798-804.

[7] Nassar M, Abdelazim IA. Pre-puncture ultrasound guided epidural insertion before vaginal delivery. Journal of Clinical Monitoring and Computing. 2015; 29: 573-577.

[8] Li H, Kang Y, Jin L, Ma D, Liu Y, Wang Y. Feasibility of ultrasoundguided lumbar epidural access using paramedian transverse scanning with the needle in-plane: a comparison with paramedian sagittal scanning. Journal of Anesthesia. 2020; 34: 29-35.

[9] Park S, Yoo S, Kim WH, Lim Y, Bahk J, Kim J. Ultrasound-assisted vs. landmark-guided paramedian spinal anaesthesia in the elderly: A randomised controlled trial. European Journal of Anaesthesiology. 2019;
36: 763-771.

[10] Debiec J, Conell-Price J, Evansmith J, Shafer SL, Flood P. Mathematical modeling of the pain and progress of the first stage of nulliparous labor. Anesthesiology. 2009; 111: 1093-1110.

[11] Polónia Valente R, Santos P, Ferraz T, Montenegro N, Rodrigues T. Effect of obesity on labor duration among nulliparous women with epidural analgesia. The Journal of Maternal-Fetal \& Neonatal Medicine. 2020; 33: 2195-2201.

[12] Shmueli A, Salman L, Orbach-Zinger S, Aviram A, Hiersch L, Chen R, et al. The impact of epidural analgesia on the duration of the second stage of labor. Birth. 2018; 45: 377-384.

[13] Poma S, Scudeller L, Verga C, Mirabile G, Gardella B, Broglia F, et al Effects of combined spinal-epidural analgesia on first stage of labor: a cohort study. The Journal of Maternal-Fetal \& Neonatal Medicine. 2019; 32: 3559-3565.

[14] Luo Y, Zhang D, Zheng J, Yang Q, An X, Chen Y, et al. Suitable depth of epidural puncture in nulliparous pregnant woman. Cell Biochemistry and Biophysics. 2015; 71: 875-880.

[15] Vallejo MC, Phelps AL, Singh S, Orebaugh SL, Sah N. Ultrasound decreases the failed labor epidural rate in resident trainees. International Journal of Obstetric Anesthesia. 2010; 19: 373-378.

[16] Grau T, Leipold R, Conradi R, Martin E, Motsch J. Ultrasonography and peridural anesthesia. Technical possibilities and limitations of ultrasonic examination of the epidural space. Der Anaesthesist. 2001; 50: 94-101.

[17] Lim YC, Choo CY, Tan KTJ. A randomised controlled trial of ultrasoundassisted spinal anaesthesia. Anaesthesia and Intensive Care. 2014; 42: 191-198.

[18] Wang Y, Ying H, Zhang W, He R, Lin J. Application of ultrasoundguided epidural catheter indwelling in painless labour. Journal of Pakistan Medical Association. 2020; 70: 45-50.

How to cite this article: Jindi Jiang, Yujie $\mathrm{Xu}$, Mingfeng He. Real-time ultrasound-guided vs. anatomical landmarkguided paramedian epidural anesthesia in overweight parturients undergoing analgesic labor: a randomized controlled trial. Signa Vitae. 2021;17(6):66-73. doi:10.22514/sv.2021.218. 